"Rondiconti del Circolo Matomatico di Palermo," Tomo xurr., Fasc. 1, 2; 1899.

"Bulletin of the Amorican Mathematical Society," 2nd Series, Vol. v., No. 6 ; Now York, Murch, 1809.

"Monatshefte fïr Mathematik und Physik," Jahrgang x., Pt. 2 ; Wien, 1898.

"Bullotin des Scionces Mathímatiques," Tome xxmr., Férrier, 1899 ; Paris.

"Rendiconto dell' Accadomia delle Scienze Fisiche e Mutematiche," Serie 3, Vol. จ., Frвc. 2, 3 ; Napoli, 1899.

" $\Lambda$ tti delln renle $\Lambda$ ccademia dei Lincoi-Rendiconti," Sem. 1, Vol. virr., Fasc. 4, 5 ; Roma, 1899.

"Berichto über dio Vorhnndlungen dor Königl. Sïchs. Gosellschnft der WiseenBchaften zu Leipzig," I., 1899.

"Nyt Tidsskrift for Matematik," $\Lambda$, $\Lambda$ argang rx., Nr. 6-8 ; Copenhagen, 1898.

"Jahrbuch über dio Fortschritte der Mathomatik," Bd. xxvir., Jahrgang, 1896, IIoft 3 ; Berlin, 1899.

Note on the Characteristic Invariants of an Asymmetric Optical System. By T. J. I'A. BRомwicr. Received March 15th, 1899. Cornmunicated $\Lambda$ pril 13̈th, 1899. Received, in revised form, May 17th, 1899.

1. The object of this note is to apply the method of the characteristic function, as treated by Maxwell and Larmor,* to deduce the invariants of an nsymmetric optical system. These have been very fully worked out by Prof. Sampson in a recent communication to the Socicty, + using a continuation of methods due to Gauss.

The direct application of the usual form for the reduced path from one point to another leads to very complicated algebra in calculating the invariants; and it appears advantagcous to uso a modificd form. The form I adopt is found by applying the Hamiltonian method of reciprocation, as employed in the genernl equations of dynamics; or, rather, Routh's modified form of the transformation, in which some of the coordinates are not transformed. The following is a short account of the theory.

- Proc. Lond. Mtath. Soc., Vols. I7., xx., xxmr.

† Ibid., Vol. xxix. 
2. Suppose $V$ represents the reduced path from a point $(x, y, z)$ in a medium of refractive index $\mu_{1}$ to $\left(x_{2}, y_{2}, z_{2}\right)$ in a sccond medium of index $\mu_{2}$. By the ordinary theory of the characteristic function the direction-cosines of the ray from the first point to the second are given by

$$
\begin{aligned}
& \mu_{1} l_{1}=-\frac{\partial V}{\partial x_{1}}, \quad \mu_{9} l_{2}=+\frac{\partial V}{\partial x_{9}} ; \\
& \mu_{1} m_{1}=-\frac{\partial V}{\partial y_{1}}, \quad \mu_{2} m_{2}=+\frac{\partial V}{\partial y_{2}} ; \\
& \mu_{1} n_{1}=-\frac{\partial V}{\partial z_{1}}, \quad \mu_{2} n_{9}=+\frac{\partial V}{\partial z_{3}} .
\end{aligned}
$$

It will be convenient to use the notation

$$
(\alpha, \beta, \gamma)=\mu(l, m, n),
$$

suffixes being attached as required. Then let

$$
U=\left(\alpha_{2} x_{2}+\left(\beta_{2} y_{2}\right)-\left(a_{1} x_{1}+\beta_{1} y_{1}\right)-V\right.
$$

and $U$ is to be expressed in terms of $a_{1} \beta_{1} z_{1}, a_{2} \beta_{2} z_{2}$. Forming the complete differential of $U$, we see that

$$
d U=\left(x_{2} d a_{2}+y_{2} d \beta_{2}\right)-\left(x_{1} d a_{1}+y_{1} d \beta_{1}\right)-\left(\frac{\partial V}{\partial z_{1}} d z_{1}+\frac{\partial V}{\partial z_{2}} d z_{2}\right),
$$

the coefficients of $d x_{1}, d y_{1}, d x_{n}, d y_{2}$ being all zero by definition of $a_{1}, \beta_{2}$, $a_{2}, \beta_{2}$. Hence we find the equations

$$
\begin{array}{ll}
x_{1}=-\frac{\partial U}{\partial a_{1}}, & x_{2}=+\frac{\partial U}{\partial \alpha_{2}} ; \\
y_{1}=-\frac{\partial U}{\partial \beta_{1}}, & y_{2}=+\frac{\partial U}{\partial \beta_{2}} ; \\
\gamma_{1}=+\frac{\partial U}{\partial z_{1}}, & \gamma_{2}=-\frac{\partial U}{\partial z_{2}}
\end{array}
$$

To ensure perfect symmetry we should have used the complete Hamiltonian transformation, but the above form has becn cmployed with a view to the special application required in this paper.

We can at once calculate the effect of a change of origins, provided that the now points are not outside the media $\mu_{1}, \mu_{2}$. If this condition is satisfied, the directions of the ray are not affected by the change ; so, supposing $z_{1}, z_{2}$ changed to $z_{1}+\zeta_{1}, z_{2}+\zeta_{2}$, respectively, we shall have that the new valuc of $U$ is

$$
U+\gamma_{1} \zeta_{1}-\gamma_{2} \zeta_{2} \text {. }
$$


This is obtained by applying Taylor's theorem and observing that $\gamma_{1}, \gamma_{3}$ are independent of $z_{1}, z_{2}$.

3. I now proceed to the consideration of the special case of thin pencils; it is known that, if each $z$ be measured along the central ray of the pencil, the approximate value of $V$ may be arranged in the form

$$
V=V_{0}+V_{8} \text {, }
$$

where suffixes indicate the order of the terms in $x_{1}, y_{1}, x_{2}, y_{2}$, and the coefficients will be functions of $z_{1}, z_{2}$; the terms rejected contain cubes and higher powers of $x_{1}, y_{1}, x_{2}, y_{2}$.

$$
\text { It is now seen that } \quad U=-V_{0}+V_{2} \text {, }
$$

when expressed in terms of $a_{1}, \beta_{1}, z_{1}, \alpha_{2}, \beta_{2}, z_{2}$; for, by Euler's theorem,

$$
x_{1} \frac{\partial V}{\partial x_{1}}+y_{1} \frac{\partial V}{\partial y_{1}}+x_{8} \frac{\partial V}{\partial x_{8}}+y_{8} \frac{\partial V}{\partial y_{8}}=2 V_{3},
$$

to our degree of approximation. Also the terms of most importance in determining the shape of the pencil are the quadratic terms, and for these

$$
U_{2}=V_{9} \text {. }
$$

Next, we have

$$
\begin{gathered}
\gamma_{1}^{2}=\mu_{1}^{2}-\left(a_{1}^{2}+\beta_{1}^{2}\right), \\
\gamma_{1}=\mu_{1}-\frac{1}{2}\left(a_{1}^{2}+\beta_{1}^{2}\right) / \mu_{1},
\end{gathered}
$$

and thus

approximately. Similarly,

$$
\gamma_{\mathrm{g}}=\mu_{\mathrm{g}}-\frac{1}{2}\left(a_{2}^{2}+\beta_{\mathrm{g}}^{2}\right) / \mu_{\mathrm{g}}
$$

Hence the change in $U_{8}$ due to moving the origins is

$$
\frac{1}{2}\left[\left(a_{2}^{2}+\beta_{8}^{2}\right) \zeta_{8} / \mu_{2}-\left(a_{1}^{2}+\beta_{1}^{2}\right) \zeta_{1} / \mu_{1}\right] .
$$

Comparing this expression with the complicated forms given by Lnrmor * for the transformation of $V_{\mathbf{g}}$ from one pair of origins to a second pair, it will be seen that we shall be able to detect the invariants of $U_{3}$ more easily than those of $V_{8}$. Further, the invariants of $U_{3}$ will be found to present themselves more naturally than those of Prof. Sampson's scheme of coefficients, which expresses $x_{1}, y_{1}, a_{1}, \beta_{1}$ in terms of $x_{2}, y_{2}, \alpha_{2}, \boldsymbol{\beta}_{2}$.

On the other hand, it must be said that in my experience it is usually ersier to calculate Prof. Sampson's scheme for a given optical system than to find either $V_{9}$ or $U_{2}$. In fact, I had used this as the best method of solving certain types of optical questions for some time before the publication of Prof. Sampson's paper. 
4. Another argument for the use of $U_{2}$ is to be found in the fact that the coefficients of $V_{2}$ will become infinite for the values $z_{1}=0, z_{3}=0$ if the origins be conjugate points of the optical system; that is, if a pencil proceeding from one origin have one of its focal lines at the other origin after passing through the system. Thus the approximations involved in the expression for $V$ would be untenable; and such a pair of origins cannot be used in that analysis. The truth of this statement may be seen from Larmor's paper." Now it is in some cases convenient to use origins of this nature; and this was the reason that originally led to my performing the Hamiltonian transformation.

To illustrate the behaviour of $U_{2}, V_{2}$ in this case we may calculate them for direct incidence on a symmetrical instrument. By the ordinary theory we have

$$
\begin{aligned}
& -\mu_{1} x_{1}=u_{1} a_{1}+f_{1} a_{2}, \quad-\mu_{1} y_{1}=u_{1} \beta_{1}+f_{1} \beta_{2}, \\
& +\mu_{2} x_{2}=f_{2} a_{1}+u_{2} a_{2}, \quad+\mu_{2} y_{3}=f_{2} \beta_{1}+u_{2} \beta_{2},
\end{aligned}
$$

where $f_{1}, f_{2}$ are the focal lengths of the instrument (connected by the equation $f_{1} / \mu_{1}=f_{2} / \mu_{2}$ ), and $u_{1}, u_{2}$ are the distances of the origins from the corresponding principal focal planes, to be taken positive when the origins are outside these planes. We then have

$$
\begin{array}{r}
2 U_{2}=\left(u_{1} / \mu_{1}\right) a_{1}^{2}+\left(f_{1} / \mu_{1}+f_{2} / \mu_{2}\right) a_{1} a_{2}+\left(u_{2} / \mu_{2}\right) a_{2}^{2} \\
+a \text { symmetrical term in } \beta, \\
2 V_{2}=\left[\left(\mu_{1} u_{2}\right) x_{1}^{2}+\left(\mu_{1} f_{2}+\mu_{2} f_{1}\right) x_{1} x_{2}+\left(\mu_{2} u_{1}\right) x_{2}\right] /\left(u_{1} u_{2}-f_{1} f_{2}\right) \\
+a \text { symmetrical term in } y,
\end{array}
$$

by using the theorems

$$
2 U_{2}=2 V_{3}=\left(a_{2} x_{2}+\beta_{2} y_{2}\right)-\left(\alpha_{1} x_{1}+\beta_{1} y_{1}\right)
$$

Examining these expressions, it is clear that $\nabla_{2}$ will be useless when $u_{1} u_{2}=f_{1} f_{8}$, or when the origins are conjugate foci of the system. On the other hand, $U_{2}$ may always be employed unless $f_{1}, f_{2}$ be infinite, or $u_{1}, u_{2}$ be infinite; in these cases the instrument will be telescopic (that is, parallel incident rays will be parallel at emergence) and the general theory must be modified to some extent.

- Cf. Sections 10 and 14 in his paper (Vol. xx.). 
5. We proceed to consider some deductions from the form of $U_{2}$. The most general form of a quaternary quadric is

$$
\begin{aligned}
2 U_{2}=a_{1} a_{1}^{2} & +2 c_{1} \alpha_{1} \beta_{1}+b_{1} \beta_{1}^{2}+a_{2} \alpha_{2}^{2}+2 c_{2} \alpha_{2} \beta_{8}+b_{8} \beta_{2}^{2} \\
& +2 p a_{1} a_{2}+2 q \alpha_{1} \beta_{2}+2 r a_{8} \beta_{1}+2 s \beta_{1} \beta_{2} .
\end{aligned}
$$

Applying the theorem proved above (Section 2), we have

$$
\begin{aligned}
-x_{1} & =a_{1} \alpha_{1}+c_{1} \beta_{1}+p a_{2}+q \beta_{2}, \\
-y_{1} & =c_{1} a_{1}+b_{1} \beta_{1}+r a_{2}+8 \beta_{2}, \\
x_{2} & =p a_{1}+r \beta_{1}+a_{2} a_{2}+c_{2} \beta_{2}, \\
y_{2} & =q a_{1}+s \beta_{1}+c_{2} \alpha_{2}+b_{2} \beta_{2},
\end{aligned}
$$

from which we can at once express the coefficients of Prof. Sampson's scheme by solving for $x_{1}, y_{1}, a_{1}, \beta_{1}$ in terms of $x_{2}, y_{2}, a_{2}, \beta_{2}$. We see that there will be sixteen coefficients; but, as these are all expressed by the aid of ten independent constants, there must be six relations amongst the sixteen. Prof. Sampson gives twelve, and shows that the second six can be deduced from the first six. I have verified these relations in the general case, but do not reproduce the work, as the algebra is straightforward but tedious; and, further, it is sufficient to verify them for the simplest canonical form of $U_{2}$, since Prof. Sampson has shown that his relations are invariant for all axes."

6. Invariants of $U_{3}$.

By the ordinary theory of quadratics, the quantities

$$
a_{1}+b_{1}, \quad a_{1} b_{1}-c_{1}^{2}
$$

are invariants for all rotations of the first set of axes about the axis of $z_{1}$. Further, by what was proved in Section 3, a change of origin will not affect the quantities

$$
a_{1}-b_{1}, c_{1} \text {. }
$$

Thus

$$
\left(a_{1}-b_{1}\right)^{3}+4 c_{1}^{2}=\left(a_{1}+b_{1}\right)^{3}-4\left(a_{1} b_{1}-c_{1}^{2}\right)
$$

is invariant for all axes.

- It is perhnps worthy of comment that, if Prof. Sampson's relations bo written in the Jacobiun form (given in Section 7 infra), they follow, from the six conditions of the typo $\frac{\partial x_{1}}{\partial \beta_{1}}=\frac{\partial y_{1}}{\partial a_{1}}$, by direct transformation of the differential coefficients to new independent variables. We infer that, written in this form, they would be truo for a beam of finite dincnsions, not merely for a thin pencil. 
Similarly, we find the invariant

$$
\left(a_{2}-b_{8}\right)^{2}+4 c_{2}^{2}=\left(a_{2}+b_{8}\right)^{2}-4\left(a_{2} b_{2}-c_{8}^{2}\right) .
$$

Next, the terms in $p, q, r, s$ may be written

$$
\left(p a_{1}+r \cdot \beta_{1}\right) a_{2}+\left(q a_{1}+\delta \beta_{1}\right) \beta_{2} .
$$

and thus for all displacements of the second set of axes we have the invariant

$$
\left(p a_{1}+r \beta_{1}\right)^{2}+\left(q a_{1}+s \beta_{1}\right)^{2}=\left(p^{2}+q^{2}\right) a_{1}^{2}+2(p r+q s) a_{1} \beta_{1}+\left(r^{2}+s^{2}\right) \beta_{1}^{2} .
$$

From this we get two absolute invariants

$$
p^{2}+q^{2}+r^{2}+s^{2}
$$

and

$$
\left(p^{2}+q^{2}\right)\left(r^{2}+s^{2}\right)-(p r+g s)^{2}=(p s-q r)^{2} .
$$

Instead of the second of these we use its square root

$$
p s-q r \text {. }
$$

Finally, combining the two quadratic forms

$$
\begin{gathered}
a_{1} a_{1}^{2}+2 c_{1} a_{1} \beta_{1}+b_{1} \beta_{1}^{2}, \\
\left(p^{2}+q^{2}\right) a_{1}^{2}+2(p r+q s) a_{1} \beta_{1}+\left(r^{2}+s^{2}\right) \beta_{1}^{2},
\end{gathered}
$$

we find that their mutual invariant is reducible to the type

$$
\left(a_{1}-b_{1}\right)\left(p^{2}+q^{2}-r^{2}-s^{2}\right)+4 c_{1}(p r+q s)
$$

or to

$$
\left(a_{1}-b_{1}\right)(p r+q s)-c_{1}\left(p^{2}+q^{2}-r^{3}-s^{2}\right) \text {. }
$$

Neither of the forms (v.), (vi.) is the ordinary harmonic invariant of the two quadratic forms, but their relations to it can be easily put down. Calling the harmonic invariant $S$, we have

$$
S=a_{1}\left(r^{2}+s^{2}\right)+b_{1}\left(p^{2}+q^{2}\right)-2 c_{1}(p r+q s),
$$

and it will be seen that

$$
(\mathrm{v} .)=\left(a_{1}+b_{1}\right)\left(p^{2}+q^{2}+r^{2}+s^{2}\right)-2 S,
$$

and

$$
\left(v_{.}\right)^{2}+4(\text { vi. })^{2}=(\text { i. }) \times\left[(\text { iii. })^{2}-4(\text { iv. })^{2}\right] \text {. }
$$

The reason for selecting (v.) or (vi.) in preference to $S$ is that $S$ is not invariant for displacements of the origins ; only for rotations of the axes about $O z_{1}, O z_{2}$. 
In the same way as ( $\nabla$.$) and (vi.), we get the invariants$

$$
\begin{aligned}
& \left(a_{8}-b_{2}\right)\left(p^{2}+r^{2}-q^{2}-s^{2}\right)+4 c_{8}(p q+r s), \\
& \left(a_{2}-h_{2}\right)(p q+r s)-c_{2}\left(p^{2}+r^{2}-q^{2}-s^{2}\right) .
\end{aligned}
$$

These satisfy the relation

$$
(\text { vii. })^{2}+4(\text { viii. })^{9}=(\text { ii. }) \times\left[(\text { iii. })^{2}-4(\text { iv. })^{2}\right] .
$$

7. We have thus found six independent invariants of $U_{9}$, and this is a complete set for the transformations to which we are restricted. Prof. Sampson has shown that there are six, and only six, invariants of his optical scheme; and the same argument may be applied here. We have ten constants in $U_{3}$ and four degrees of freedom for the axes, namely, displacements along and rotations about the two axes of $z_{1}, z_{2}$. Hence there must be six, and only six, fundamental constants of an optical system for thin pencils.

It will be now easy to find the relations between the invariants of Prof. Sampson's scheme and those discussed in the last section. To do this, let our axes be chosen so as to make some of the coefficients zero; by rotating the axes we can make $q=0, r=0$, and, in addition, it will be convenient to make $Q=0, R=0$ if possible; we use the notation $Q, R$ for the minors of $q, r$ in $\Delta$, the discriminant of $U_{g}$. After putting $q=0, r=0$, we find

$$
\begin{aligned}
& Q=p b_{1} c_{2}+8 a_{9} c_{1}, \\
& R=p b_{2} c_{1}+s a_{1} c_{2} .
\end{aligned}
$$

Now shifting the origins distances $\mu_{1} \rho_{1}, \mu_{2} \rho_{2}$ along the axes of $z_{1}, z_{9}$ away from the instrument will change $a_{1}, b_{1}, a_{2}, b_{2}$ to the quantities $a_{1}+\rho_{1}, b_{1}+\rho_{1}, a_{2}+\rho_{3}, b_{2}+\rho_{2}$; so that it is usually possible to select the origins in such a way as to give $Q=0, R=0$. Exceptional cases will arise if $c_{1}=0, c_{3}=0$, when the conditions are satisfied for all positions of the origins; and if $p^{3}=s^{8}$, when the conditions cannot be satisfied unless $\left(a_{1}-b_{1}\right) / c_{1}=\left(a_{2}-b_{2}\right) / c_{2}$. Excluding these cases, we deduce from our expressions for $x_{1}, y_{1}, x_{2}, y_{2}$ in terms of $a_{1}, \beta_{1}$, $a_{2}, \beta_{q}$ the following scheme, of the type given by Prof. Sampson (p. 66, loc. cit.) :-

$$
\begin{aligned}
& x_{1}=-\left(a_{1} / p\right) x_{2}+\left(a_{1} a_{9} / p+c_{1} c_{2} / s-p\right) a_{2}-\left(c_{1} / s\right) y_{2}, \\
& a_{1}=(1 / p) x_{2}-\quad\left(a_{2} / p\right) a_{3}
\end{aligned}
$$


The determination of the origins by the conditions $Q=0, R=0$ will ensure the vanishing of the coefficients of $\beta_{2}$ in $x_{1}$, and of $a_{2}$ in $y_{1}$; the coefficients of $y_{2}$ in $\alpha_{1}$, and of $x_{2}$ in $\beta_{1}$, are zero in consequence of $q=0, r=0$.

We can immediately verify the twelve relations amongst the coefficients given by Prof. Sampson, contained in equations (1) ... (6), (1')... (6') of his paper. Three typical equations are

$$
\begin{aligned}
& \frac{\partial\left(x_{1}, a_{1}\right)}{\partial\left(x_{2}, a_{2}\right)}+\frac{\partial\left(y_{1}, \beta_{1}\right)}{\partial\left(x_{2}, a_{2}\right)}=1 \\
& \frac{\partial\left(x_{1}, a_{1}\right)}{\partial\left(x_{2}, y_{2}\right)}+\frac{\partial\left(y_{1}, \beta_{1}\right)}{\partial\left(x_{2}, y_{2}\right)}=0 \\
& \frac{\partial\left(x_{1}, a_{1}\right)}{\partial\left(a_{2}, \beta_{8}\right)}+\frac{\partial\left(y_{1}, \beta_{1}\right)}{\partial\left(a_{2}, \beta_{2}\right)}=0 .
\end{aligned}
$$

Of these the first two follow at once from the expressions above; and the third is verified on observing that $a_{1} a_{3} / p^{8}=b_{1} b_{8} / s^{2}$ from the conditions $Q=0, R=0$. As remarked before, the relations written in these forms will be true for a beam of any size, not only for a thin pencil.

8. In the last section we obtained a form of $U_{2}$ which contains six independent constants; or, rather, eight, with the two relations $Q=0, R=0$. This form may be called canonical, and it will be convenient to allude to it by the letter $(A)$ in order to distinguish it from another canonical form to be used later.

I find that, with this scheme, the chief invariants of Prof. Sampson's notation are

$$
\begin{aligned}
(a) & =\frac{1}{p^{2}}+\frac{1}{s^{2}}, & (d) & =c_{1}\left(\frac{1}{s^{2}}-\frac{1}{p^{2}}\right), \\
(b) & =\frac{1}{p s}, & (f) & =c_{2}\left(\frac{1}{s^{2}}-\frac{1}{p^{2}}\right), \\
\left(a c^{\prime}\right) & =\frac{a_{1}-b_{1}}{p s}\left(\frac{1}{s^{2}}-\frac{1}{p^{2}}\right), & \left(a f^{\prime}\right) & =\frac{a_{9}-b_{2}}{p s}\left(\frac{1}{p^{2}}-\frac{1}{s^{2}}\right), \\
(D) & =\frac{\left(a_{1}-b_{1}\right)^{2}}{p^{2} s^{2}}+c_{1}^{2}\left(\frac{1}{p^{2}}+\frac{1}{s^{2}}\right)^{2}, & \left(F^{\prime}\right) & =\frac{\left(a_{2}-b_{2}\right)^{2}}{p^{2} s^{2}}+c_{2}^{2}\left(\frac{1}{p^{2}}+\frac{1}{s^{2}}\right) .
\end{aligned}
$$


Further, in my notation,

$$
\begin{aligned}
\text { (i.) } & =\left(a_{1}-b_{1}\right)^{2}+4 c_{1}^{2}, & & \text { (ii.) }=\left(a_{2}-b_{2}\right)^{2}+4 c_{2}^{2}, \\
\text { (iii.) } & =p^{2}+s^{2}, & & \text { (iv.) }=p s, \\
\text { (v.) } & =\left(a_{1}-b_{1}\right)\left(p^{2}-s^{2}\right), & & \text { (vi.) }=-c_{1}\left(p^{2}-s^{2}\right), \\
\text { (vii.) } & =\left(a_{2}-b_{8}\right)\left(p^{2}-s^{2}\right), & & \text { (viii.) })=-c_{2}\left(p^{2}-s^{9}\right) .
\end{aligned}
$$

Thus we have

$$
\begin{aligned}
(a) & =(\text { iii. }) /(\text { iv. })^{2}, & (b) & =1 /(\text { iv. }), \\
(d) & =-(\text { vi. }) /(\text { iv. })^{2}, & (f) & =-(\text { viii. }) /(\text { iv. })^{2}, \\
(a c) & =(\text { v. }) /(\text { iv. })^{3}, & \left(a f^{\prime}\right) & =-(\text { vii. }) /(\text { iv. })^{8}, \\
(D) & =(d)^{2}+(\text { i. }) /(\text { iv. })^{2}, & \left(F^{\prime}\right) & =\left(f^{2}\right)+(\text { ii. }) /(\text { iv. })^{2} .
\end{aligned}
$$

The other invariants given by Prof. Sampson can be expressed in terms of these; and it is thus unnecessary to write out the complete list. The only one presenting any difficulty is $(K)$, which may be shown to be

$$
c_{1} c_{3} p s\left(\frac{a_{1} a_{9}}{p^{2}}+\frac{c_{1} c_{2}}{p s}-1\right)\left(\frac{1}{p^{2}}-\frac{1}{s^{2}}\right)^{3},
$$

the apparent want of symmetry of this being accounted for by the relation $a_{1} a_{2} / p^{2}=b_{1} b_{2} / s^{2}$. The expression for $(K)$ in terms of the other invariants is given by Prof. Sampson, but need not be written out here, as it is rather lengthy.

It will be seen that, if $(l)=0$, and $(f)=0$ in Prof. Sampson's notation; or if (vi.) $=0$, (viii.) $=0$ in the invariants above, we are brought to the exceptional cases of $c_{1}=0, c_{2}=0$, or $p=s$. If $p=s$, the reduction to the form $(A)$ is not possible unless

$$
\left(a_{1}-b_{1}\right) / c_{1}=\left(a_{2}-b_{2}\right) / c_{2} \text {. }
$$

9. Another canonical form, denoted by (B), is found by taking as the origin on each side of the system one of the points conjugate to infinity. This has some advantages, as it contains only six coefficients, which are thus all in rariants. This reduction will be always possiblo unless the system is quasi-telescopic; that is, unless rays incident parallel to the central ray emerge parallel to the emergent central ray.

With these origins we shall have that, if $x_{1}=0, y_{1}=0$, then $a_{2}=0$, $\beta_{2}=0$, provided a suitable relation be taken between $\alpha_{1}, \beta_{1}$. Hence the equations

$$
\begin{aligned}
& a_{1} a_{1}+c_{1} \beta_{1}=0 \\
& a_{1} a_{1}+b_{1} \beta_{1}=0
\end{aligned}
$$


will hold for the same ratio $a_{1}: \beta_{1}$, that is

$$
a_{1} b_{1}-c_{1}^{2}=0
$$

But by rotation of axes we can make $c_{1}=0$, and hence one of the two $a_{1}, b_{1}$ will be zero, say $b_{1}$.

In the same way we may have

$$
b_{9}=0, \quad c_{2}=0 .
$$

Thus the canonical form is given by

$$
2 U_{2}=a_{1} \alpha_{1}^{2}+a_{2} \alpha_{2}^{2}+2\left(p a_{1} \alpha_{2}+q a_{1} \beta_{2}+r \alpha_{2} \beta_{1}+s \beta_{1} \beta_{2}\right) \text {. }
$$

The second focal line of an incident pencil whose rays will emerge parallel to the central ray may be easily found. For let this be $z_{1}=-\mu_{1} \rho_{1}$, so that $\rho_{1}$ is positive when measured away from the instrument. Then we are to have that $x_{1}=\rho_{1} a_{1}, y_{1}=\rho_{1} \beta_{1}$ give $a_{2}=0$, $\beta_{2}=0$ provided a suitable relation between $\alpha_{1}, \beta_{1}$ be chosen. We find at once that $a_{1}+\rho_{1}=0$. In the same way the second focal line of an emergent pencil, whose rays are incident parallel to the central rny, is given by $a_{2}+\rho_{2}=0$.

10. With the canonical form $(B)$, we have invariants

$$
\begin{aligned}
\text { (i.) } & =a_{1}^{2}, & & \text { (ii.) }=a_{2}^{2}, \\
\text { (v.) } & =a_{1}\left(p^{2}+q^{2}-r^{2}-s^{2}\right), & & \text { (vi.) }=a_{1}(p r+q s), \\
\text { (vii.) } & =a_{2}\left(p^{2}+r^{2}-q^{2}-s^{2}\right), & & \text { (viii.) }=a_{2}(p q+r s),
\end{aligned}
$$

(iii.) and (iv.) are, of course, unaltered from their values in the general case.

Hence we have a geometrical interpretation of the invariants (i.) and (ii.); in fact (i.) $\times \mu_{1}^{2}$ is the square of the distance between the first principal focal lines of the instrument; or between the pair of lines from which a pencil must proceed in order to emerge as a parallel beam. Similarly we interpret (ii.) $\times \mu_{2}^{2}$.

With this arrangement of axes the relation between conjugate foci trkes a simple for'm. Suppose that a pencil from the point $z_{1}=-\mu_{1} \rho_{1}$ proceeds on emergence from a focal line at $z_{2}=\mu_{2} \rho_{2}$; then we shall have $x_{1}=\rho_{1} \alpha_{1}, y_{1}=\rho_{1} \beta_{1}, x_{2}=-\rho_{2} a_{2}, y_{2}=-\rho_{2} \beta_{2}$ for the same ratios

$$
a_{1}: \beta_{1}: a_{2}: \beta_{2} \text {, }
$$

or

$$
\left|\begin{array}{cccc}
a_{1}+\rho_{1} & 0 & p \\
0 & \rho_{1} & r & s \\
p & r & a_{3}+\rho_{8} & 0 \\
q & s & 0 & \rho_{g}
\end{array}\right|=0 .
$$


This gives on expansion

$$
\begin{aligned}
\rho_{1} \rho_{2}\left(a_{1}+\rho_{1}\right)\left(a_{9}+\rho_{2}\right) & -p^{9} \rho_{1} \rho_{9}-q^{2} \rho_{1}\left(a_{2}+\rho_{2}\right)-r^{9} \rho_{9}\left(a_{1}+\rho_{1}\right) \\
& -s^{3}\left(a_{1}+\rho_{1}\right)\left(a_{9}+\rho_{9}\right)+(p s-q r)^{2}=0 .
\end{aligned}
$$

Denoting by $F, G$ the points where the first principal focal lines meet the central ray; and by $F^{\prime}, G^{\prime}$ similar points for the second focal lines, we shall have, if $P, Q$ be the points $\rho_{1}, \rho_{9}$,

$$
P F=\mu_{1} \rho_{1}, \quad P G=\mu_{1}\left(a_{1}+\rho_{1}\right), \quad Q F^{\prime}=\mu_{3} \rho_{2}, \quad Q G^{\prime}=\mu_{9}\left(a_{3}+\rho_{2}\right) .
$$

Hence the relation just written becomes

$P F . P G . Q F^{\prime} . Q G^{\prime}$

$$
\begin{aligned}
& -\mu_{1} \mu_{3}\left[p^{2}\left(P F \cdot Q F^{\prime}\right)+q^{2}\left(P F \cdot Q G^{\prime}\right)+r^{2}\left(P G^{\prime} . Q F^{\prime}\right)+s^{2}\left(P G . Q G^{\prime}\right)\right] \\
& +\left(\mu_{1} \mu_{2}\right)^{2}(p s-q r)^{2}=0 .
\end{aligned}
$$

Of course all the coefficients involved may be readily put down in terms of invariants if required; but the expressions are not simple, excepting the one $(p s-q r)$ which is the invariant (iv.).* .

11. It may be remarked in connexion with the classification of optical systems given by Prof. Sampson at the end of his paper that two conditions are requisite for his class (iv.), namely, $(d)=0$ and $(f)=0$ independently ; $\dagger$ both conditions being necessary in order that this class may possess the property stated by Prof. Sampson.

This property is that the projections of a ray on two suitably chosen coordinate planes may be treated as if refracted independently of each other. Investigating this we find the two conditions $c_{1}=0, c_{2}=0$ in scheme $(A)$ and $q=0, r=0$ in scheme $(B) ;$ and then the two canonical reductions are virtually the same.

It seems possible that Prof. Sampson was led to suppose that $(d)$ could not vanish without $(f)$ by examining his scheme of simplified

- Consider a cone of rays from $P$; the emergent rays will rnark out a certain area on a plane at any point $Q$, drawn at right angles to the central ray. Wo may then define $d$, the apparcnt distance of $Q$ as seen from $P$, by the equation

$$
\left.d^{2}=\text { (area at } Q\right) /\left(\text { solid angle of cone at } l^{\prime}\right) \text {. }
$$

It is easy to show that the left-hand side of the lust equation, beginning with $P F . P G . Q F \cdot Q G^{\prime}$ is equal to $\mu_{2}^{2} d^{2}\left(p s-q{ }^{\prime}\right)$.

+ Prof. Sampson states, without proof, that the invariant (d) cannot vanish in systems of finite curvatures without making $(f)$ zero as well. 
invariants (p. 67 of his paper). Here $(d)=0$ certainly appears to involve $(f)=0$; but this is only the case either if $\varpi_{1}^{2}=w_{q}^{2}$, or if $k=0$. Neither of these conditions enables us to satisfy the fundamental property stated above; the true condition is not easily expressed in terms of the coefficients which are there employed; for, if the true condition be satisfied, $k=\infty$, while $k \varpi_{1}, k \varpi_{2}$ are finite.

\section{The Theorem of Residuation, Noether's Theorem, and the Riemann-} Roch Theorem. By F. S. MACAULAY. Received March 28th, 1899. Read April 13th, 1899.

The following paper is $\mathrm{a}$ re in the nature of an essay than of a rigorous investigation. Its object is to advance and explain general notions rather than to give incontrovertible proofs of all the statements made. Sections I. and II. contain a discussion of the most general aspect of the T'heorem of Residuation, and lead, in Section III., to an analytical and generalized interpretation of results previously deduced geometrically. (Proc. Lond. Math. Soc., Vol. xxix., pp. 673-695.)

The fundamental idea of the paper is a very simple one, viz., that the whole intersection of two given curves $C_{l}, C_{m}$ at a common point $A$ may be resolved into an equivalent aggregate, $a$, of simple points, no matter how complex the forms of the two curves at $A$ may be. These a points are brought into evidence analytically by the fact that they supply a independent linear equations for the coeflicients of a general algebraic curve of sufficiently high order. It is highly probable that the $a$ equations could alway's be written in such a form, and arranged in such order, that each new equation, interpreted in connexion with those which precede it and apart from those which succeed it, expresses the condition that the curve passes through a new point, or, more strictly, possesses a property equivalent to that of passing through a new point. Such an arrangement is not, however, attempted per se in the paper.

The fundamental notion of a complex point being equivalent to an aggregate of simple points is in no sense a novel one; but its very simplicity has been considered as liable to lead to erroneous 